\title{
Design a calorimeter system for the Muon Collider experiment
}

\section{Sestini, ${ }^{a, *}$ P. Andreetto, ${ }^{a}$ A. Gianelle, ${ }^{a}$ D. Lucchesi, ${ }^{a, b}$ L. Buonincontri, ${ }^{a, b}$ \\ D. Zuliani, ${ }^{a, b}$ M. Casarsa, ${ }^{c}$ N. Bartosik, ${ }^{d}$ N. Pastrone, ${ }^{d}$ I. Sarra, ${ }^{e}$ M. Swiatlowski ${ }^{f}$ and \\ M. Valente ${ }^{f}$}

a INFN Sezione di Padova, Padova, Italy

${ }^{b}$ University of Padova, Padova, Italy

${ }^{c}$ INFN Sezione di Trieste, Trieste, Italy

${ }^{d}$ INFN Sezione di Torino, Torino, Italy

e INFN Laboratori Nazionali di Frascati, Frascati, Italy

${ }^{f}$ TRIUMF, Vancouver, Canada

E-mail: lorenzo.sestini@pd.infn.it

\begin{abstract}
A muon collider is being proposed as a next generation facility. This collider would have unique advantages, since clean events as in electron-positron colliders are possible, and high collision energy as in hadron colliders could be reached due to negligible beam radiation losses. The beaminduced background, produced by the muon decays in the beams and subsequent interactions, reaches the interaction region and the detectors and presents unique features and challenges with respect to other machines. As an example, a diffused flux of photons and neutrons passes through the calorimeter system, which thus requires a design to avoid this substantial background. In this talk an overview of the calorimetry at the Muon Collider is given, with a particular focus on the reconstruction and measurement of hadronic jets, that are studied with the full simulation of the detector. RD for new calorimeter technologies, developed specifically for the Muon Collider, will be also presented.
\end{abstract}

\footnotetext{
*** The European Physical Society Conference on High Energy Physics (EPS-HEP2021), ***

*** 26-30 July $2021 * * *$

*** Online conference, jointly organized by Universität Hamburg and the research center DESY ***
}

\footnotetext{
${ }^{*}$ Speaker
} 


\section{Introduction}

The Muon Collider is one of the most promising proposals for future accelerators. It puts together the advantages of having relatively clean events as in lepton colliders, and the possibility to reach high collision energy as in hadron colliders, since bremsstrahlung is very limited.

However the Muon Collider environment is not so clean as one can expect, since the presence of the beam-induced background (BIB), produced by the decay of muons and subsequent interactions, may pose limitations to the physics performance [1]. Although the BIB can be partially mitigated by a proper design of the machine-detector-interface, e.g. by using two shielding tungsten nozzles in the detector region [3], it poses requirements on the detector development [3]. In this document the development of a calorimeter system that could be suited for the Muon Collider environment is discussed.

\section{Calorimeter requirements}

In the following study the BIB is simulated at $\sqrt{s}=1.5 \mathrm{TeV}$ by using the MARS15 package [4], while the detector is simulated using a custom branch of ILCSoft [5]. The baseline calorimeter system is that designed by the CLIC collaboration [6]: an electromagnetic calorimeter (ECAL) with 40 layers of tungsten (W) absorber and silicon ( $\mathrm{Si}$ ) pad sensors of $5 \times 5 \mathrm{~mm}^{2}$ area, and a hadron calorimeter (HCAL) with 60 layers of steel absorber and plastic scintillating tiles of $30 \times 30 \mathrm{~mm}^{2}$ area. The interaction of the BIB with the calorimeters is simulated and it is compared with the simulation of a reference signal: the Higgs boson decaying to two b-quarks.

As shown in figure 1 (left), BIB hits are out-of time with respect to the signal coming from the bunch-crossing: for this reason we believe that the time measurement is crucial to get rid of a large part of the background. In fact, an acquisition time window of \pm 250 ps can remove most of the $\mathrm{BIB}$, preserving the signal. We assume that this window can be achieved with a time measurement resolution of about $80 \mathrm{ps}$.
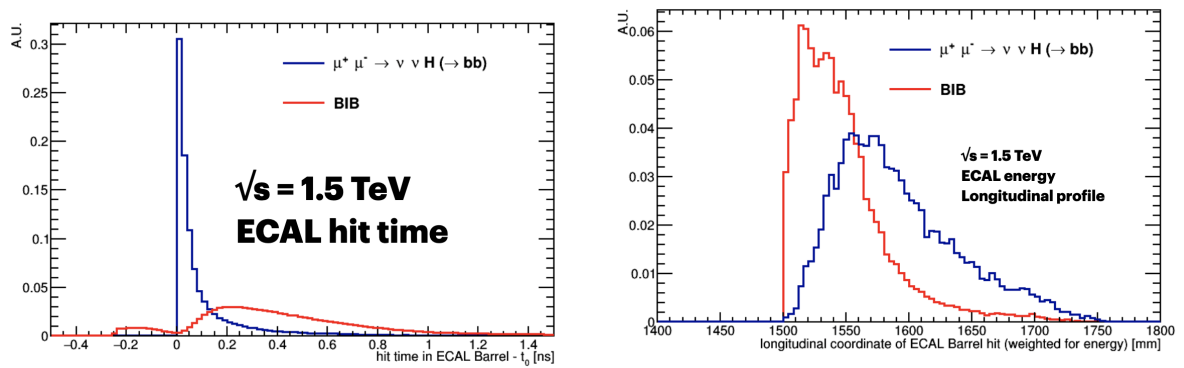

Figure 1: Left: time of the ECAL barrel hits with respect to the bunch crossing time, for BIB and $H \rightarrow b \bar{b}$. Right: energy distribution of ECAL barrel hits as a function of the distance with respect to the beam axis, for BIB and $H \rightarrow b \bar{b}$.

In figure 1 (right) The longitudinal profile of the energy released by the BIB is compared with the signal. The profiles are quite different, since the BIB deposits most of the energy in the first layers, while the signal releases energy also in deeper layers. It is clear that a longitudinal segmentation 
of the calorimeter can be useful to identifay the fake showers produced by the combinatorial of the BIB hits.

\section{Proposal for a new technology: Crilin}

In this section we study a technology alternative to the W-Si sampling calorimeter for the ECAL barrel. Although the calorimeter designed by the CLIC collaboration is highly segmentated, the cost can be very high, and the electronic read-out system for so many channels is not a trivial task. In this section the proposed technology is a semi-homogeneous crystal calorimeter $\left(\mathrm{PbF}_{2}\right)$, where Cherenkov light is read by silicon photomultipliers. This calorimeter has been called Crilin [7]. $\mathrm{PbF}_{2}$ crystals has good light yield ( 3 photo-electrons per $\mathrm{MeV}$ ) and fast signals ( $300 \mathrm{ps}$ for muons, $50 \mathrm{ps}$ for pions). They are also radiation hard and relatively cheap. The Crilin ECAL barrel design for the Muon Collider consists of five layers with $40 \mathrm{~mm}$ thick, and $10 \times 10 \mathrm{~mm}^{2}$ of cell area. Real cell prototypes has been built at the National Laboratory of Frascati (Rome, Italy), and their properties and currently under validation in dedicated test beams.

In order to assess the physics performance of the Crilin ECAL barrel, this has been implemented in the full simulation of the Muon Collider experiment. In figure 2, energy distributions of hits produced by the BIB and by the signal are shown, for different calorimeter regions (central and forward) and different layers. Since the BIB and signal distributions are different, energy thresholds are applied as a function of the layer and of the region. Thresholds are obtained as $E_{T H}=E_{B I B}+2 \sigma_{B I B}$, where $E_{B I B}$ is the average energy of the BIB distribution, and $\sigma_{B I B}$ is the standard deviation. The performance is evaluated on objects of primary interest for Muon Collider
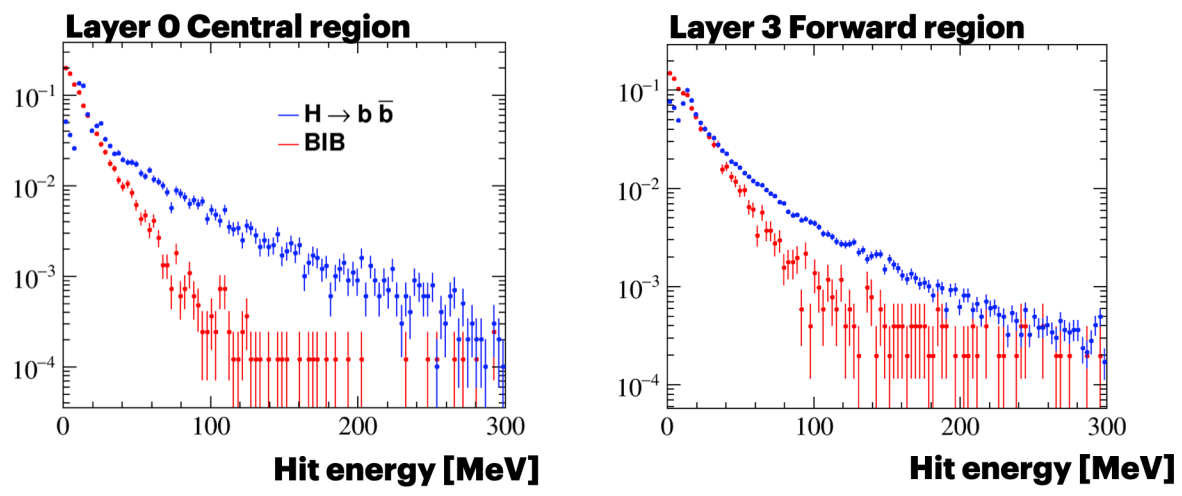

Figure 2: Left: distribution of the hit energy for BIB and $H \rightarrow b \bar{b}$ in the first layer and in the central region $(|z|<500 \mathrm{~mm}$, where $z$ is the coordinate parallel to the beam axis). Right: distribution of the hit energy for BIB and $H \rightarrow b \bar{b}$ in the third layer and in the forward region $(|z|>500 \mathrm{~mm})$.

physics: hadronic jets. A Particle Flow algorithm that involves both calorimeter and tracker is employed for jet reconstruction [8]. This algorithm is not yet fully optimized, but it is useful for comparing the performance of different detector systems. A simulation sample of $b$-jets has been used for this purpose. The jet reconstruction efficiency and jet $p_{T}$ resolutions are presented in figure 3. It can be seen that the performance is similar in the two cases, where the W-Si or the BIB ECAL barrel are used. 

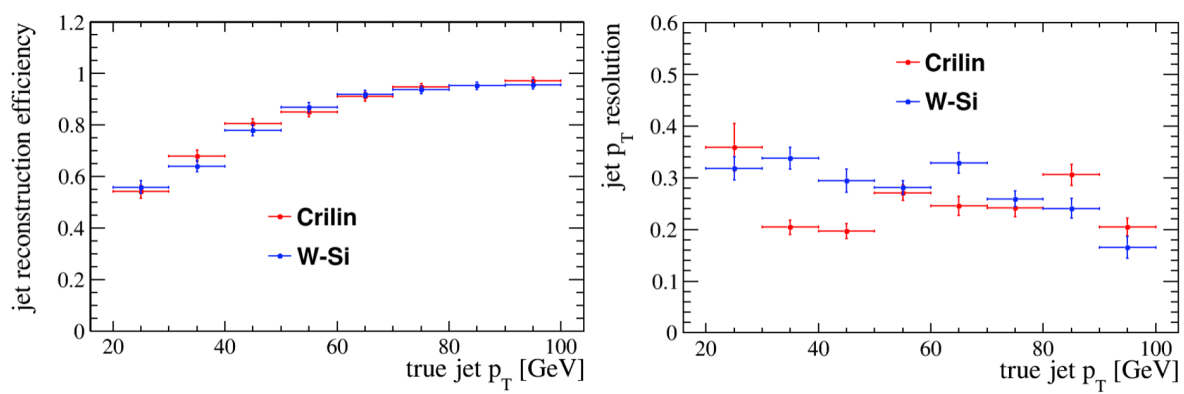

Figure 3: Left: jet reconstruction efficiency as a function of the jet $p_{T}$, obtained by using the Crilin ECAL barrel and the W-Si ECAL barrel. Right: jet $p_{T}$ resolution as a function of the jet $p_{T}$, obtained by using the Crilin ECAL barrel and the W-Si ECAL barrel.

\section{Conclusions}

The beam-induced background (BIB) at a Muon Collider poses specific requirements on the detector development. It has been demonstrated with the simulation that precise timing measurement $\left(<100\right.$ ps resolution) and longitudinal segmentation are important features. A 5-layers $\mathrm{PbF}_{2}$ calorimeter, called Crilin, is proposed as technology for the ECAL barrel. By using the simulation, the performance on the jet reconstruction obtained with Crilin has been compared with the one obtained with a highly segmented W-Si sampling calorimeter proposed by the CLIC collaboration. It has been found that the two calorimeters have similar jet reconstruction efficiencies and $p_{T}$ resolutions. A possible explanation is that, since the performance is limited by the BIB, a highly segmented calorimeter is not really needed at a Muon Collider, and a 5-layer calorimeter can be sufficient. Moreover Crilin may cost a factor 10 less than W-Si. Further studies are needed to reach a conclusive statement on which is the best technology for the Muon Collider calorimeter.

\section{References}

[1] Muon Accelerator Program, https://map.fnal.gov/

[2] N.V. Mokhov and S.I.Striganov, Phys. Procedia 37 (2012) 2015

[3] N. Bartosik et al, 2020 JINST 15 P05001

[4] N.V. Mokhov and C.C. James, Fermilab-FN-1058-APC (2018)

[5] https://github.com/iLCSoft

[6] CLIC collaboration, CERN-2012-003

[7] A. Cemmi et al, https://arxiv.org/abs/2107.12307

[8] L. Sestini et al, Jet Reconstruction performance at Muon Collider with Beam-induced Background, APS April Meeting 2021 